\title{
Volume Rendering Dark Matter Simulations Using Cell Projection and Order-Independent Transparency
}

\author{
Oleg Igouchkine, Nick Leafł, and Kwan-Liu Ma ${ }^{\ddagger}$ \\ University of California, Davis
}

\begin{abstract}
Dark matter simulations, performed using N-body methods with a finite set of tracer particles to discretize the initially uniform distribution of mass, are an invaluable method for exploring the formation of the universe. Definining a tetrahedral mesh in phase spacewith the tracer particles at initialization serving as vertices-yields a more accurate density field. At later timesteps, the mesh selfintersects to an enormous degree, making pre-sorting impossible. Kaehler et al [2012] visualize the mesh using cell projection, but their method requires order-independent compositing, which limits its flexibility. Our work renders the mesh using state of the art order-independent transparency (OIT) techniques to composite fragments in correct depth order. This also allows us to render variables other than density, such as velocity. We implement a number of OIT optimizations to handle the high depth complexity (on the order of $10^{7}$ depth layers for $2 \times 10^{9}$ particles) of the data. Our performance measurements show near-interactive framerates for our hybrid renderer despite the large number of depth layers.
\end{abstract}

Keywords: Scientific Visualization, Volume Rendering, Intersecting Mesh, Order-Independent Transparency, GPU Acceleration

Concepts: $\bullet$ Computing methodologies $\rightarrow$ Scientific visualization; Rendering; Volumetric models; •Applied computing $\rightarrow$ Astronomy;

\section{Introduction}

Dark matter makes up the majority of our universe by mass but, by definition, cannot be directly observed. Astronomers are able to infer the existence of dark matter through its gravitational influence on observable matter. By lining up real observations with the output from N-body dark matter simulations, scientists are able to create a verifiable and explorable model of the formation of the universe. Performing the simulations on a large enough scale for good resolution poses significant computation and analysis challenges.

$\mathrm{N}$-body dark matter simulations represent mass using a number of tracer particles, each of which represents a mass initially distributed uniformly in some local neighborhood, which can be treated as a collisionless fluid. Visualization of the tracer particles is an integral analysis tool, but traditional methods suffer from a number of

\footnotetext{
*email: oigouchkine@ucdavis.edu

†email: njleaf@ucdavis.edu

†email: ma@cs.ucdavis.edu
}

Permission to make digital or hard copies of all or part of this work for personal or classroom use is granted without fee provided that copies are not made or distributed for profit or commercial advantage and that copies bear this notice and the full citation on the first page. Copyrights for components of this work owned by others than the author(s) must be honored. Abstracting with credit is permitted. To copy otherwise, or republish, to post on servers or to redistribute to lists, requires prior specific permission and/or a fee. Request permissions from permissions@acm.org. (C) 2016 Copyright held by the owner/author(s). Publication rights licensed to ACM.

SA '16 Symposium on Visualization ,, December 05 - 08, 2016, , Macao ISBN: 978-1-4503-4547-7/16/12

DOI: http://dx.doi.org/10.1145/3002151.3002163 artifacts. Kernel-based density estimation tends to under-represent mass in low-density regions. Resampling to a regular grid and interpolating density from that alleviates some problems, but introduces new ones in the form of a tradeoff between the loss of highfrequency detail and excessive memory use.

Recently, multiple works [Shandarin et al. 2012; Abel et al. 2012] have computed the density field using a phase-space tetrahedral mesh defined between tracer particles. Each tetrahedron is assumed to have identical mass, uniformly distributed throughout its volume. The mesh connectivity is defined in phase-space at the first timestep, and remains constant thereafter. The continuous nature of the mesh yields much better spatial resolution than particle-only methods. Kaehler et al. [2012] demonstrate artifact-free rendering of low-density regions using the phase-space mesh via a cell projection method. Though this method produces high quality images at a fairly good speed, it can only compute the integral of the 3D density field along each ray, and not the full volume rendering integral. The same work also covered resampling the phase-space mesh to regular grid, but this was subject to the aforementioned detail versus memory tradeoff.

In this work, we study methods to combine the flexibility afforded by full volume rendering with the accuracy achieved by cell projection of the phase-space mesh. Pre-sorting the mesh is not an option due to its large degree of self-intersection-we have observed regions with thousands of intersecting tetrahedra in a typical $2 \mathrm{~m}$ particle dataset. We propose per-ray fragment-sorting methods as a solution. Traversing a fragment list in depth order, the density can be increased at tetrahedron front faces, and decreased at back faces to yield the correct density along the ray. The large amount of overlapping depth layers (115k for the $2 \mathrm{~m}$-particle dataset) still poses a significant problem under this scheme, and we describe several methods to improve the rendering speed. The summarized contributions of this work are:

- a view-independent approach to visualizing the phase-space mesh,

- a rendering approach that is extensible to the velocity field and other derived statistics,

- an approach for order-independent-transparency suitable for hundreds of thousands of depth layers,

- and a screen-space hybrid approach which mixes exact and approximate rendering based on depth complexity.

\section{Related Work}

\subsection{Field Estimation from Particle Data}

The Lagrangian model of fluid simulation uses a number of tracer particles to predict the behavior of the entire fluid. Each tracer particle is a discretization representing some mass (typically equal for all tracers) of real particles. Most visualization and analysis techniques require a continuous representation of the density field at some point. Properly computing the continuous density is an area of ongoing research. 
Smoothed Particle Hydrodynamics (SPH) [Monaghan 1988] approximates density by convolving each tracer particle with some kernel to spread the representative mass over a local volume. The density field at a point is then computed by summing the contributions from neighboring tracer particles. SPH enables direct visualization from the particles by splatting them to the screen [Hopf and Ertl 2003] using a constant or adaptive kernel, and this approach can be scaled up to very large datasets with good performance [Fraedrich et al. 2009]. Direct particle splatting has also been used to compare differences between simulation runs with similar initial conditions [Haroz et al. 2008]. SPH can also be used to indirectly visualize the density field via resampling it to a regular grid [Cha et al. 2009; Fraedrich et al. 2010; Popov et al. 2011]. An alternative to SPH for density estimation is to create a Voronoi or Delaunay tessellation from which the density field can be interpolated [Bernardeau and van de Weygaert 1996]. One way to avoid sorting particles is to stochastically render particles as fully-opaque to multiple render targets and average the results [Zhao et al. 2015].

Recently, multiple works [Shandarin et al. 2012; Abel et al. 2012] have defined an unstructured mesh using the initial phase-space positions of the tracer particles, where each cell represents a constant mass of matter. The phase-space mesh provides a more accurate density field approximation than kernel-based methods, and can be used to improve simulation accuracy [Hahn et al. 2013]. The phasespace mesh can also be used to create extremely high-quality, artifact free visualizations. [Kaehler et al. 2012] used the initial particle ordering to define a memory-efficient tetrahedral mesh. The tetrahedra can then be splatted to the screen to compute a per-pixel integral of the density field.

\subsection{Tetrahedral Rendering}

Numerous methods have been proposed to render unstructured tetrahedral meshes. Shirley and Tuchman introduced Projected Tetrahedra (PT) [Shirley and Tuchman 1990] to compute the rendering contribution of each tetrahedra as it is splatted to the screen. Numerous improvements have been made to the original algorithm since then, such as removing artifacts [Kraus et al. 2004], adding hardware acceleration [Wylie et al. 2002], and accelerating the render-order sort via the GPU [Maximo et al. 2010]. PT has been shown to scale well for distributed rendering of large-scale data [Ma and Crockett 1997]. Tetrahedral meshes have also been rendered directly via raycasting [Weiler et al. 2003; Espinha and Celes 2005].

One major distinction between the phase-space mesh and most tetrahedral meshes is that the phase-space mesh can overlap to an effectively arbitrary degree. [Shih et al. 2014] presents a rendering method for multiple overlapping grids, both unstructured and structured. However, the meshes in their data only overlap locally, and only to a small degree (at most four). The phase-space mesh of a cosmological dark matter simulation can self-intersect to a much larger degree, with a single tetrahedra potentially intersecting with thousands of others. Every tetrahedra overlapping a given point must be accounted for to correctly compute density. The number of intersections makes either per-sample neighbor lookup or vieworder sorting infeasible, which would be required for direct raycasting or projection. [Kaehler et al. 2012] was able to circumvent this problem by computing the order-independent density integral for each ray.

\subsection{Order-Independent Transparency}

Our proposed method bears a number of similarities to OrderIndependent Transparency (OIT) methods, which aim to provide robust rendering of partially transparent meshes without the need for pre-sorted primitives. Depth peeling [Everitt 2001] is a robust OIT method which works well with GPU hardware, but it requires one geometry pass for every one or two [Bavoil and Myers 2008] depth layers. The phase space mesh has $6 N$ tetrahedra for an $N$-particle mesh, with thousands of potential depth layers, which would lead to an extremely large number of depth peeling passes in practice.

The A-buffer [Carpenter 1984] enables OIT by creating a list of fragments for each pixel, or Pixel Linked List (PLL). This approach has received renewed interest due to the availability of GPU features which enable hardware PLLs [Yang et al. 2010], and multiple extensions to improve memory use and locality have since been proposed [Maule et al. 2012; Vasilakis and Fudos 2012; Lindholm et al. 2015]. Work has also been done on adapting OIT methods to handle depth-fighting [Vasilakis and Fudos 2013]. One useful tweak to PLLs is to, instead of keeping every fragment, only keep a fixed-size list of fragments per-pixel which closely approximates the exact color [Salvi et al. 2011]. [Maule et al. 2011] provides a thorough survey of transparency techniques for raster-based rendering.

\section{The Phase-Space Mesh}

The mass density distribution of N-body cosmological dark matter simulations is most commonly computed-in the visualization field, at least-by applying a smoothing kernel to the tracer particles. Previous work [Shandarin et al. 2012; Abel et al. 2012] has shown that the distribution can be more accurately represented by a tetrahedral mesh with its vertices at the particles, and its connectivity defined by a tetrahedralization of particle positions in the initial time step. Importantly, the connectivity remains constant across the entire simulation. The mass represented by the tracer particles is evenly distributed amongst the tetrahedra. Since the initial masss distribution is completely uniform, each tetrahedron contains an identical amount of mass. The density for a single tetrahedron can be computed by dividing its constant mass by its volume for the current timestep. The density of a point in 3D is the superposition of density from all tetrahedra covering that point.

Many N-body dark matter simulations initially place the tracer particles on a regular cubic grid. The position of each particle in such a layout can be computed from its ID with a simple mapping, which makes it easy to also compute the neighbors of each particle. If particle IDs are consistent for the entire simulation, then the original neighborhood, and thus the phase-space tessellation, can be directly computed at any timestep. We tesselate the initial particle grid using the same method as [Kaehler et al. 2012]. The grid is divided into cubic cells, with tracer particles at the cell vertices. Each cubic cell is tessellated by six tetrahedra. The cell tessellation was chosen so that each tetrahedron has identical volume, and so that edges and faces between cells are consistent. Because the mesh connectivity is constant and the same tessellation is used for each cubic cell, the mesh can be efficiently computed as neccessary and does not need to be explicitly stored.

\section{Methodology}

The core of our method is based on cell projection, but the nature of the phase-space mesh makes pre-sorting intractable. Subdividing the tetrahedra into non-intersecting convex sub-volumes would incur an enormous memory cost and produce arbitrary polyhedra with per-element masses. We expect that the benefits of a sortable mesh would be heavily outweighed by the additional computational complexity and memory cost.

Instead, we opted for an OIT-based approach that was compatible with the existing data structure. We generate one vertex for each 
tracer particle, and use a geometry shader to generate the tetrahedral faces for the tessellation. The faces are projected to the screen, creating an unordered list of fragments for each pixel. Once the fragments are sorted in depth order, then density can be recovered at any point along the ray by keeping track of which tetrahedra encompass the current position. To compute ray color, we traverse the list from front to back, adding to the current density when we encounter a ray's front face, and subtracting when we encounter a back face.

The downside of the OIT-plus-cell-projection approach is that the fragment lists can grow quite large. Much of our work has been dedicated to improving the performance of fragment list storage and rendering, whether by reducing the number of fragments that must be stored, or by implementing fast sorting methods. Figure 1 shows images comparing the exact rendering, bin approximation, and hybrid method, as well as an image showing the cutoff region (described more in Section 4.4).

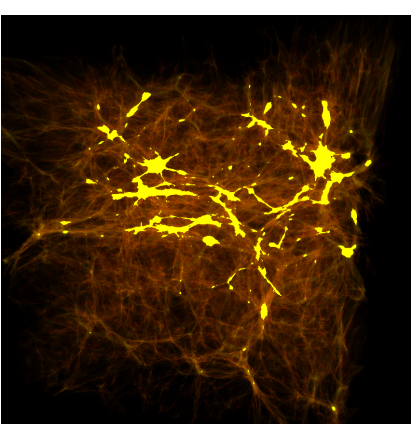

(a) Exact Approach

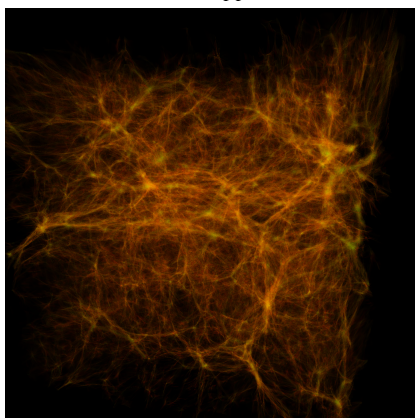

(c) Hybrid Method

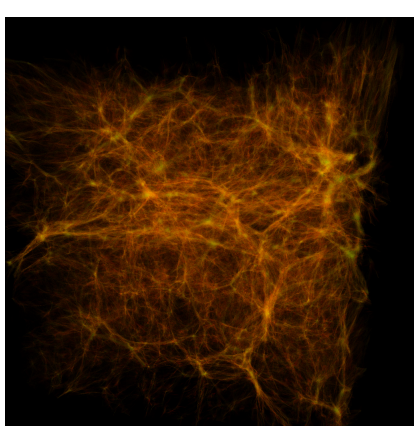

(b) Bin Approximation

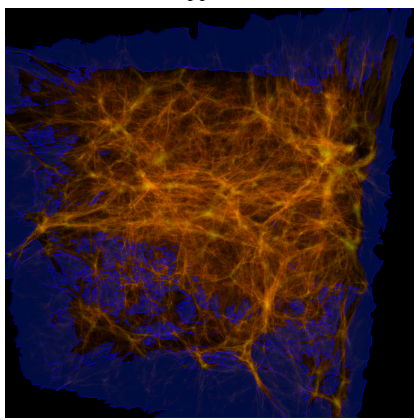

(d) Cutoff region highlighted
Figure 1: Comparison of volume rendering results using our proposed methods. Attempting to render and composite all fragments (1a) fails to complete in high depth-complexity regions (yellow, $16.9 \%$ of rendered pixels). The bin approximation renders quickly, but introduces some noise. The hybrid method uses the exact method where it can (39.8\%), and switches to the binning method for pixels with high depth complexity (60.2\%). The cutoff region (blue) shows which pixels are rendered by the exact method.

\subsection{Exact Method}

Our direct approach handles the data through cell-projection, using the front faces of each tetrahedron to indicate the ray entry point and the back faces to indicate the exit point. Dut to the large amount of intersection in the mesh, a sort operation on each face is impossible without additional geometry; we instead incorporate state-of-theart order-independent transparency methods to sort faces during the final ray integration.

In our base implementation, we utilize a dynamic fragment buffer [Vasilakis and Fudos 2013], a method which precomputes the number of fragments in each pixel location then allocates sections in a global buffer for each pixel. We use two channels for representing the fragments as they are generated; we store the fragment depth in one channel, and the precomputed change in density in the other. In the ray-integration step, we utilize both per-pixel array optimization (ppAO) and per-pixel depth peeling (ppDP) [Lindholm et al. 2015]. These methods reduce the amount of local memory used in each fragment shader invocation by separating the rendering of lightweight and heavyweight pixels, and looping over the global memory with a small local array. The output at each step of the implementation is a short, sorted list of fragments. Each fragment list is guaranteed to be in the correct order relative to the other segments. The sorted fragments can then be used to build ray segments with associated density and length.

To create and integrate these ray segments, we use counters for the current density and distance from origin. For each loop, we use the current distance as the ray start point, and the distance of the next fragment as the ray end point; the current density is used for the ray, then updated by adding the next fragment's density contribution. The final algorithm is shown in algorithm 1, where $\rho$ is the density, $d$ is the distance to the camera, and $R\left(\rho, d, d_{f}\right)$ is the opacity-corrected transfer function for a distance $d_{f}-d$.

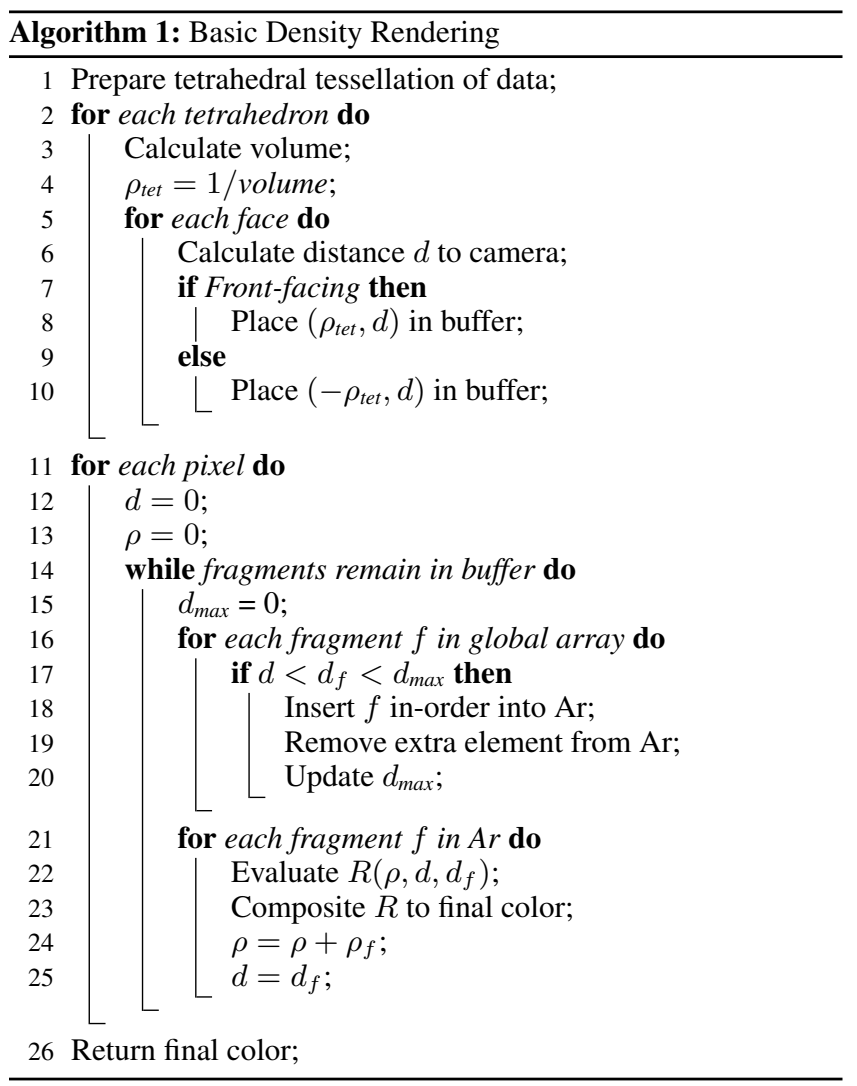

\subsection{Optimizations to OIT}

While state-of-the-art OIT methods improve the efficiency of rendering significantly, we have found several optimizations to the base implementation which further increase performance. The first is to resolve coplanar fragments early during the per-pixel depth peel, which eliminates the possibility of z-fighting issues and reduces the number of iterations necessary for correct resolution. The second optimization is the shrinking of the global fragment array 
while rendering, allowing a theoretical speedup of up to two times. Our final optimization is the use of a bucket sort to reduce the number of fragments which need to be sorted or iterated over.

\subsubsection{Early Coplanarity Resolution}

The use of per-pixel depth peeling as introduced in [Lindholm et al. 2015] can introduce issues due to z-fighting when fragments occur with identical depth values. Since our datasets exhibit a very high amount of intersection, we find that this is a significant issue. Some of the existing approaches to solving this issue include the use of per-fragment IDs to ensure a unique ordering; a more complete discussion may be found in [Maule et al. 2012]. However, these solutions assume that coplanar fragments have an ordering and therefore do not consider the possibility that coplanar fragments may be combined in a unique manner, thus degrading performance.

Because we use fragments to represent the change in density at a spatial location, we find that two fragments sharing the same depth value may have their density values combined additively with no influence on the final solution; if two such fragments exist in the list of fragments to be processed, then each would add its contribution to the final density while producing a ray segment with no length, which would result in no contribution from that ray segment to the final solution. Because ppDP uses an insertion sort to process incoming fragments, we implement our merge during the comparison stage of the insertion sort. Because this effectively increases the number of fragments which are stored in local memory without increasing the storage requirement, this efficiently reduces the number of iterations over the full fragment list.

\subsubsection{Fragment Array Reduction}

Per-pixel depth peeling creates a tradeoff by reducing the local array size, and thus local storage size, for each fragment shader invocation at the cost of needing to iterate over the global array of fragments multiple times. We propose that by shrinking the list of fragments to be looked over dynamically, we can improve reduce the number of passes by up to a factor of two.

Our implementation of this reduction consists of updating the head pointer to the start of the fragment buffer every pass, by incrementing it by the size of the local array $n$. We avoid the need to move up all elements as in a traditional resize operation, because we hold all elements which would be removed in the local array; if a new fragment displaces an element in the local array, we store the value in the local array back into global memory, thus preventing any unresolved elements being lost. While this method increases the $\mathrm{I} / \mathrm{O}$ traffic, we have found that it improves performance in practice.

When this approach is combined naively with early coplanarity resolution, the solution is no longer correct because the local array will hold combined fragments, and simply inserting these into the global memory allows them to be combined multiple times. Therefore, when a fragment is merged into the local array, its value in global memory must be set to zero; furthermore, if $m$ merged elements occur before the local array has been filled, it is necessary to increment the head pointer by an additional $m$ elements.

Algorithmically, these two optimizations can be represented as replacing lines 16 through 20 of algorithm 1 with algorithm 2 .

\subsubsection{Bucket Sort}

Order-independent transparency methods have a runtime which typically depends superlinearly on the number of fragments in each buffer, whether due to the cost of a sort operation or due to the need to iterate over these extra fragments. We therefore propose the use

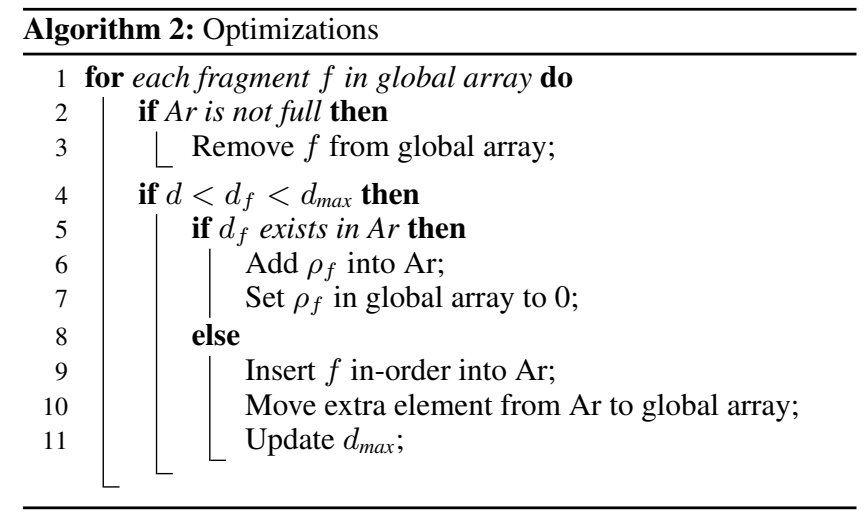

of a bucket sort to break up the fragments by depth, helping to reduce the cost of these operations.

The primary change of this method is to use $b$ buffers per pixel; in the case of the dynamic fragment buffer, this causes memory overhead due to the need for additional head pointers. In the preliminary counting stage, we count the number of fragments falling into each bucket instead of into each pixel; this requires an additional depth comparison. The primary performance penalty comes from the prefix sum calculation for creating the offsets into the dynamic fragment buffer, which must now sum over $b$ times as many elements. During the final resolve step, we proceed through each bin in sequence, keeping the density and distance counters between each bin.

\subsection{Bin Approximation}

Even with our improvements to performance, we find that the exact OIT solution does not render at an interactive speed. We therefore propose an approximation method based on merging nearby fragments and a bucket-sort optimization. The original merging algorithm only looks at fragments which are coplanar, ensuring that the final result is correct; however, we propose that merging fragments within some small distance $\epsilon$ will only remove ray fragments with a small contribution to the result, allowing us to sacrifice a small amount of accuracy for performance. We can then propose to merge all fragments within a small depth range, which results in a small change to the distance for each fragment, and the further removal of small ray segments.

With this extension, we can obtain a very large performance improvement by defining these depth ranges early and using atomic image add operations to merge fragments; this is equivalent to creating bins along the viewing direction and summing the density into each bin. Through this approach, we remove the dependence of the final resolve step on the number of fragments in each pixel, thus allowing many fragments to be rendered at interactive rates.

The basic algorithm is presented in algorithm 3 .

\subsection{Hybrid Rendering}

While the binned approximation method is capable of giving highly interactive results, the quality of the resulting image is decreased compared to the exact solution through the introduction of noise. Because the performance of the exact method is dependent on the number of fragments at a given pixel, we can switch on a per-pixel basis between using the exact method and the bin approximation. As switching between light and heavy pixels is already done for the per-pixel array optimization (ppAO) in the exact method, and 


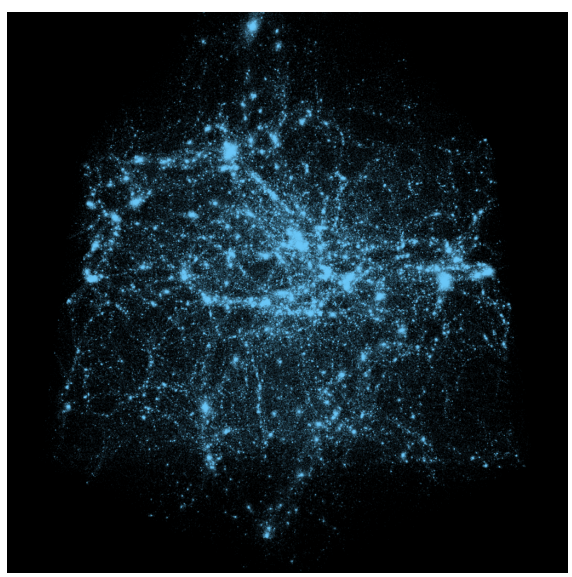

(a) Particle Splatting

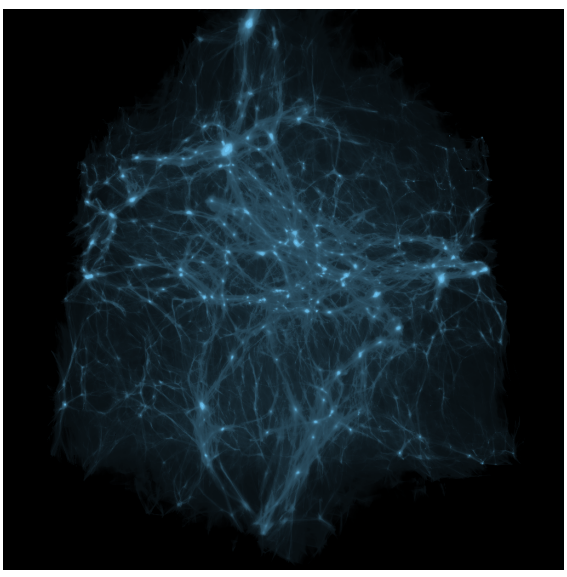

(b) Density Integral

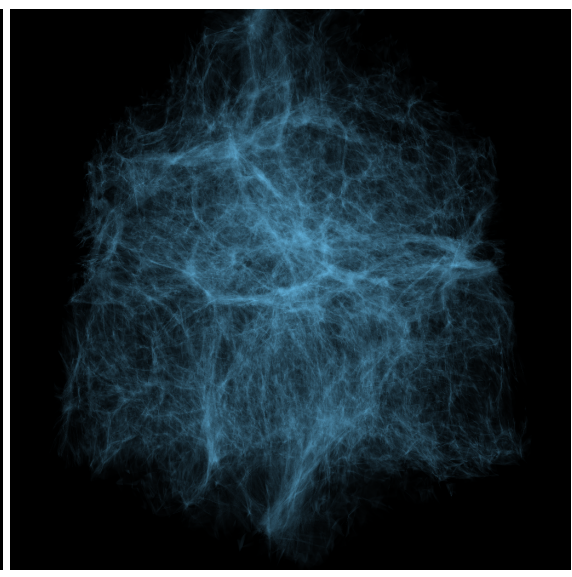

(c) Hybrid

Figure 2: Comparison with prior rendering methods. Standard, kernel-based particle splatting 2 a shows unrealistic clumpiness, and underrepresents low-density regions. The Density Integral method $2 b$ provides better representation of the density in sparse regions, unveiling the filaments between dark matter halos. Our hybrid method $2 c$ gives more flexibility in assigning the transfer function, and renders with correct occlusion to aid in understanding the complex $3 D$ structures.

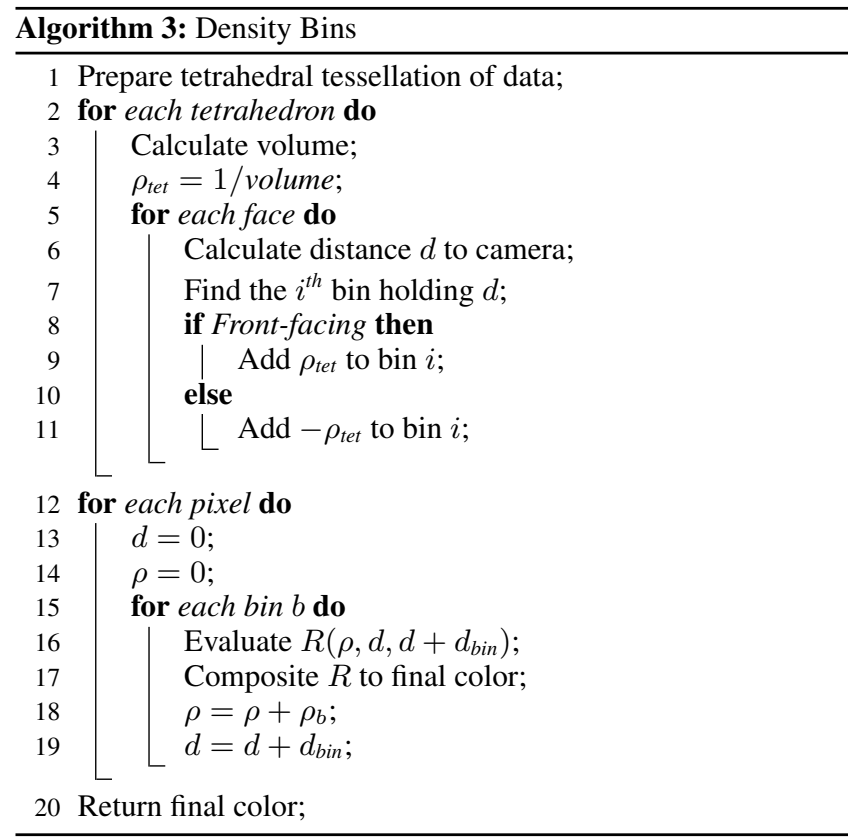

calculating the number of fragments per pixel is necessary to store them in a dynamic frame buffer, performing this switch uses functionality which is already present in the pipeline.

While switching between light and heavy pixels is already present for $\mathrm{ppAO}$, the approximation and exact methods require different actions to be undertaken during the first pass rendering of the tetrahedra. While a naïve approach may undertake two passes, we use a single pass with the fragment shader switching between storing fragments or merging them into bins.

Because we know the maximum number of elements which are to be sorted during the final ray composition, we find that not all optimizations which we use in the pure exact method are helpful. In particular, the bucket sort optimization does not improve performance at high numbers of bins, as the overhead costs overcome the benefit that is otherwise attained. Furthermore, because we use a small fragment count as the switching point, we can fit all fragments into the fragment shader's local memory, and we do not use the per-pixel depth peeling (ppDP) approach nor do we use the optimizations intended for that method.

\subsection{Velocity Field Data}

In addition to simply rendering the density field, we have attempted to extend our approach to render other types of information about the particles, including data which varies across an individual tetrahedron. In particular, we extend this approach to render velocity field data, which is attained as a linear interpolation of the velocities of the four vertices for each tetrahedron. At a given point in space, the total velocity is given by the sum of density-weighted velocities of all tetrahedra surrounding the point.

To determine the velocity contribution of each tetrahedron, the velocity information of each particle is passed to the GPU. In the geometry shader, the velocities and positions of all four points are multiplied by the density, then passed to the fragment shader. Furthermore, as each face of the tetrahedron is generated, we also generate the barycentric screen-space coordinates of the remaining vertex. In the fragment shader, for each front-face, we use the interpolated velocity of its three corners to find the entry velocity; we then use the barycentric coordinates to find which backface the fragment belongs to, and use the vertices to find the distance along the ray and the velocity at the exit position. We then store both the velocity at the entry point and the change in velocity per unit distance in each element of the dynamic fragment buffer. For each backface, the reverse is done, using the frontface to calculate the change in velocity; we also negate the velocity and change in velocity before storage.

For compositing the ray segments, we accumulate both the velocity and the change in velocity at each ray step, and use the distance from the previous step to adjust the velocity by the accumulated change in velocity. The gives us the correct velocity at each step, by considering the difference in velocity across every tetrahedron in addition to the contribution of the front- and back-faces. 


\section{Results}

In this section, we compare the images produced using rayintegrated density to those produced using a density integral and using particle splatting, and compare the image quality of our bin approximation, exact rendering, and the hybrid method. Settings used for the exact rendering were 2000 buckets for the bucket-sort optimization, and a local array size of 16 for ppDP. Furthermore, we render the image using tiles of $64 \times 64$ pixels to prevent GPU lockup caused by excessive render time. For the binned approximation, we use 2000 bins. For the hybrid method, we use 40 buckets for the bucket-sort optimization, 2000 bins for the approximation, and switch when any single bin holds more than 120 fragments. We use different transfer functions for the density-projection and particle splatting to allow for better comparison.

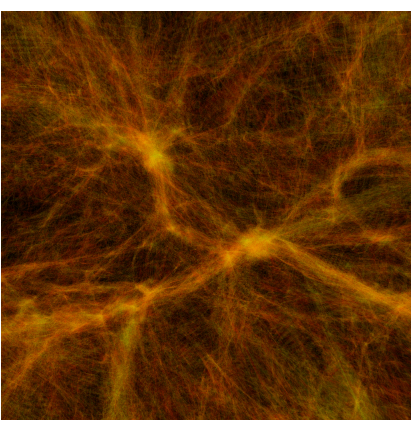

(a) 500

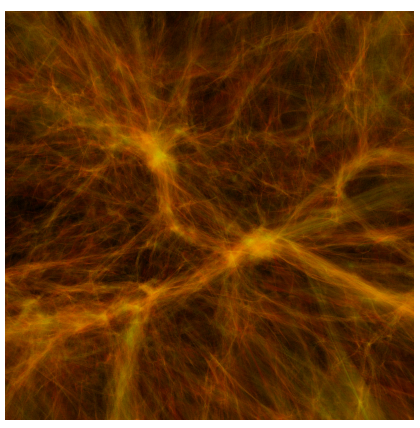

(b) 2000
Figure 3: Effect of bin amount on the quality of the bin approximation method. Lower bin counts (3a) cause high-frequency, specklelike artifacts which are most obvious in low-frequency areas. These artifacts are significantly reduced by increasing the bin count ( $3 b)$.

\subsection{Stanford Dark Sky Simulations}

Our first example is the Stanford Darksky dataset, which was the subject of the 2015 SciVis contest, at the latest available timestep. The original simulation run contained 1.1 trillion particles spread over a 38-billion light-year cube. The decimated dataset that we visualize here contains 2 million particles, regularly spaced on a $128^{3}$ grid at the initial timestep. The data is preprocessed to renumber index IDs according to the tetrahedral mesh subdivision. We show a comparison between our hybrid approach, Particle Splatting, and Density Integral in Figure 2.

We found that the exact solution failed to render at pixels with extremely high fragment counts, as calls to OpenGL which took too long triggered the hardware watchdog timer. While the bucket sort optimization is intended to reduce the number of fragments which must be dealt with at once, there were still bins which contained too many fragments; we introduced a cutoff region where any pixel with a bin containing more than 3020 fragments did not get rendered, and we represent this region using yellow.

The bin approximation introduces high-frequency noise into the final rendering, which is worse when the number of bins used is smaller. Figure 3 shows the differences when varying the number of bins. By comparison, in regions where the exact approach is successful, features are sharp. Because we use the same configuration for the bin approximation whether it is run alone or in the hybrid method, the regions where the bin approximation is used are not visibly different between the two approachs; furthermore, because the exact solution does not introduce error, the regions where the exact solution is used will not be visibly different from the exact solution.

We also show a comparison of the results achieved when rendering velocity and when rendering density in Figure 4 . For both renders we use the bin approximation with a bin count of 1000, due to the increased memory cost of rendering velocity. Furthermore, the transfer function was modified between both renders to achieve more comparable results, but colors used were identical. We note that the velocity field generally has a value even in low-density regions, which causes a more opaque render and less noise in the result. Furthermore, in the density rendering high-valued regions are more uniformly spread through the results, while in the velocity rendering they are more clearly concentrated. This viewpoint also shows an advantage of our method; due to occlusion cues, it is possible to see where regions are further back, and two overlapping regions of interest do not merge together.

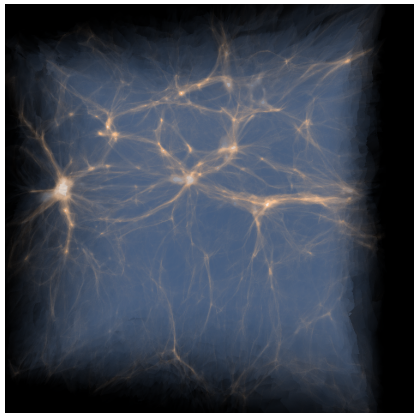

(a) Velocity

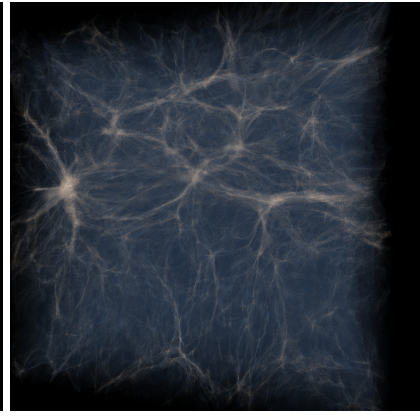

(b) Density
Figure 4: Comparison of density and velocity renderings. Matter near the center of dark matter halos tends to have higher velocity, so velocity magnitude and density tend to be correlated. Comparing the two may yield insight into halo formation or simulation limitations.

\subsection{Warm Dark Matter Dataset}

Our second example is a Warm Dark Matter dataset. This dataset contains approximately 17 million particles, initially placed on a $258^{3}$ grid. This dataset used particle IDs already suited for our method, but it did not contain velocity data. We show a comparison of our method with the density-integral method in figure 5 .

The transfer function used between the two images was not identical, to allow better comparison between the two methods. While the density-intergal method is capable of more clearly showing the high-density region at the center of the volume, it cannot display the boundary regions of the surrounding volume, since these boundary regions are added into the total density projection. Our method is also capable of displaying additional features, such as the region colored light blue. With density projection, it is not clear whether the region is of interest alone or due to the interaction between different sections.

\subsection{Performance}

Our performance tests are all performed using an NVidia GeForce Titan X with 12 GB of Video Memory, and a $3.5 \mathrm{GHz}$ Intel Core i7 5930 six-core CPU with 8 GB of RAM. The first ten frames were discarded to allow for settling time and cache propagation before collecting the actual run time. Images for performance were taken at 700x700 resolution, using the same transfer functions and 


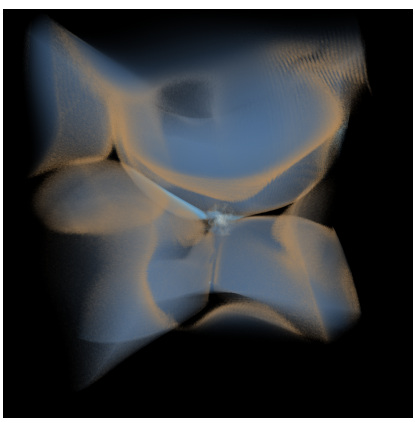

(a) Hybrid Approach

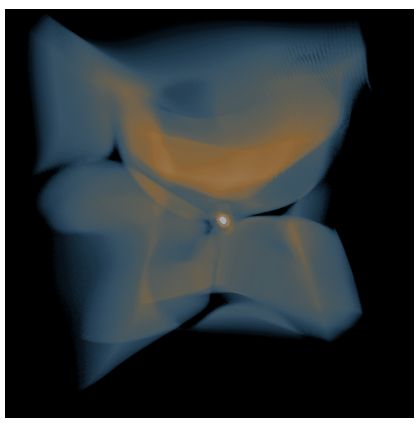

(b) Density-Integral Projection

Figure 5: Comparison of our approach with a similar approach, Density-Integration through cell projection. Note that we are capable of displaying boundaries and other features which cannot be shown using the full density integral.

settings for each method as in figure 2. Table 1 shows the performance of the presented methods, as well as the performance of Density-Integral projection [Kaehler et al. 2012].

Figure 6 shows render time versus fragment threshold for the hybrid method on the Dark Sky data. The figure shows that the render time increases superlinearly with the threshold. We did not test values larger than 120 fragments due to degrading performance.

\begin{tabular}{|l|l|l|l|l|}
\hline Dataset & $\begin{array}{l}\text { Exact } \\
\text { Method }\end{array}$ & $\begin{array}{l}\text { Bin } \\
\text { Approx }\end{array}$ & Hybrid & $\begin{array}{l}\text { Density- } \\
\text { Integral } \\
\text { Projection }\end{array}$ \\
\hline \hline $\begin{array}{l}\text { Stanford } \\
\text { Dark-Sky }\end{array}$ & $217.1 \mathrm{~s}$ & $0.569 \mathrm{~s}$ & $1.394 \mathrm{~s}$ & $0.111 \mathrm{~s}$ \\
\hline $\begin{array}{l}\text { Warm } \\
\text { Dark- } \\
\text { Matter }\end{array}$ & $74.1 \mathrm{~s}$ & $0.578 \mathrm{~s}$ & $1.32 \mathrm{~s}$ & $0.434 \mathrm{~s}$ \\
\hline
\end{tabular}

Table 1: Render time comparison of proposed methods (Exact Method, Bin Approximation, Hybrid) and a state-of-the-art method (Density-Integral Projection).

\section{Conclusion and Future Work}

We have demonstrated a method for volume rendering the phasespace mesh using cell projection and OIT. Our method is viewindependent, but still captures the fine details exposed by the meshbased density estimation approach. Our approach also allows us to visualize variables which are order-dependent along the ray, such as the velocity. Since the velocity field is interpolated from the vertices of each tetrahedron, rendering any other property which can be defined at the locations of tracer particles is straightforward.

The large degree of self-intersection (on the order of $N / 10^{3}$ ) and resulting high depth complexity (on the order of $N / 10^{2}$ ) required specialized order-independent transparency methods. Our results show that, despite the number of depth layers, we were still able to achieve near-interactive framerates with our hybrid approach. Our approach may be applicable to other data with extreme depth complexity where partial transparency is required.

One major area of future work is to improve the scalability of our method. Because it requires a large amount of data per-pixel (either for PLLs or bins) and there is no way to pre-sort the mesh, extending the method to cluster-level parallelism is not straightforward. Subdividing the mesh at intersection lines is also problematic. With

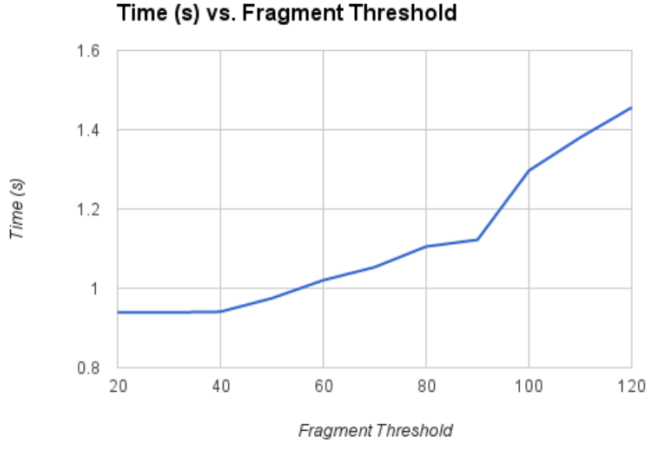

Figure 6: Render time vs. fragment threshold for Stanford Dark Sky data. The fragment threshold determines when the hybrid renderer switches from the exact to the approximate binned approach. Higher thresholds improve the quality of the result, at the cost of increased render times.

no bounds on particle position between the first and last dataset, the amount of geometry required for a sortable mesh would grow superlinearly with the number particles. One approach for solving this issue would be to create a fixed-size approximation of the function for each pixel as, say, a piecewise linear function.

Another area for future work is in the intergration of the velocity field. While we sample the velocity correctly at each face, our method does not completely consider the change in velocity between faces. Because we know that this change is linear, we propose that some form of integration along the transfer function, such as through pre-integration techniques, would allow for a more accurate rendering of the velocity field.

\section{Acknowledgements}

This research is supported in part by the U.S. National Science Foundation via grants NSF IIS-1528203 and NSF IIS-1320229, and the U.S. Department of Energy through grants DE-FC0212ER26072 and DE-SC0012610.

\section{References}

Abel, T., Hahn, O., And Kaehler, R. 2012. Tracing the dark matter sheet in phase space. Monthly Notices of the Royal Astronomical Society 427, 1 (Nov.), 61-76.

BAVOIL, L., AND MYers, K. 2008. Order independent transparency with dual depth peeling. NVIDIA OpenGL SDK, 1-12.

Bernardeau, F., And VAn de Weygaert, R. 1996. A new method for accurate estimation of velocity field statistics. Monthly Notices of the Royal Astronomical Society 279, 2, 693 711.

CARPEnTER, L. 1984. The A -buffer, an Antialiased Hidden Surface Method. In Proceedings of the 11th Annual Conference on Computer Graphics and Interactive Techniques, ACM, New York, NY, USA, SIGGRAPH '84, 103-108. 
Cha, D., Son, S., And Ihm, I. 2009. GPU-Assisted High Quality Particle Rendering. Computer Graphics Forum 28, 4 (June), $1247-1255$.

Espinha, R., And Celes, W. 2005. High-Quality HardwareBased Ray-Casting Volume Rendering Using Partial PreIntegration. In XVIII Brazilian Symposium on Computer Graphics and Image Processing (SIBGRAPI'05), 273-280.

EVERITt, C. 2001. Interactive order-independent transparency. White paper, nVIDIA 2, 6, 7.

Fraedrich, R., Schneider, J., And Westermann, R. 2009. Exploring the Millennium Run - Scalable Rendering of LargeScale Cosmological Datasets. IEEE Transactions on Visualization and Computer Graphics 15, 6, 1251-1258.

Fraedrich, R., Auer, S., And Westermann, R. 2010. Efficient High-Quality Volume Rendering of SPH Data. IEEE Transactions on Visualization and Computer Graphics 16, 6 (Nov.), 1533-1540.

HAhn, O., ABEL, T., AND KAEHLER, R. 2013. A new approach to simulating collisionless dark matter fluids. Monthly Notices of the Royal Astronomical Society 434, 2 (Sept.), 1171-1191.

Haroz, S., Ma, K. L., and Heitmann, K. 2008. Multiple Uncertainties in Time-Variant Cosmological Particle Data. In 2008 IEEE Pacific Visualization Symposium, 207-214.

Hopf, M., AND ERTL, T. 2003. Hierarchical Splatting of Scattered Data. In Proceedings of the 14th IEEE Visualization 2003 (VIS'03), IEEE Computer Society, Washington, DC, USA, VIS '03, 57-

Kaehler, R., Hahn, O., And Abel, T. 2012. A Novel Approach to Visualizing Dark Matter Simulations. IEEE Transactions on Visualization and Computer Graphics 18, 12 (Dec.), 2078-2087.

Kraus, M., Qiao, W., AND Ebert, D. S. 2004. Projecting tetrahedra without rendering artifacts. In IEEE Visualization, 2004, 27-34.

Lindholm, S., Falk, M., Sundn, E., Bock, A., Ynnerman, A., AND RoPINSKI, T. 2015. Hybrid Data Visualization Based on Depth Complexity Histogram Analysis. Computer Graphics Forum 34, 1 (Feb.), 74-85.

MA, K.-L., And Crockett, T. W. 1997. A Scalable Parallel Cell-projection Volume Rendering Algorithm for Threedimensional Unstructured Data. In Proceedings of the IEEE Symposium on Parallel Rendering, ACM, New York, NY, USA, PRS '97, 95-ff.

Maule, M., Comba, J. L. D., Torchelsen, R. P., And BasTOS, R. 2011. A survey of raster-based transparency techniques. Computers \& Graphics 35, 6 (Dec.), 1023-1034.

Maule, M., Comba, J. L. D., Torchelsen, R., And Bastos, R. 2012. Memory-Efficient Order-Independent Transparency with Dynamic Fragment Buffer. In 2012 25th SIBGRAPI Conference on Graphics, Patterns and Images, 134-141.

Maximo, A., Marroquim, R., AND Farias, R. 2010. Hardware-Assisted Projected Tetrahedra. Computer Graphics Forum 29, 3 (June), 903-912.

Monaghan, J. J. 1988. An introduction to SPH. Computer Physics Communications 48, 1 (Jan.), 89-96.
Popov, U., Heitmann, K., Ahrens, J., Habib, S., And Pang, A. 2011. The Evolution of Multistreaming Events in the Formation of Large Scale Structures. In Proceedings of the 2011 IEEE Pacific Visualization Symposium, 207-14.

Preston, A., Ghods, R., Xie, J., Sauer, F., Leaf, N., Ma, K.-L., Rangel, E., Kovacs, E., Heitmann, K., And HABIB, S. 2016. An integrated visualization system for interactive analysis of large, heterogeneous cosmology data. In 2016 IEEE Pacific Visualization Symposium (PacificVis), IEEE, $48-55$.

Salvi, M., Montgomery, J., And Lefohn, A. 2011. Adaptive Transparency. In Proceedings of the ACM SIGGRAPH Symposium on High Performance Graphics, ACM, New York, NY, USA, HPG '11, 119-126.

Shandarin, S., Habib, S., And Heitmann, K. 2012. Cosmic web, multistream flows, and tessellations. Physical Review D 85 , 8 (Apr.), 083005

Shih, M., Zhang, Y., Ma, K.-L., Sitaraman, J., And MAVRIPLIS, D. 2014. Out-of-core visualization of time-varying hybrid-grid volume data. In 2014 IEEE 4th Symposium on Large Data Analysis and Visualization (LDAV), 93-100.

Shirley, P., AND Tuchman, A. 1990. A Polygonal Approximation to Direct Scalar Volume Rendering. In Proceedings of the 1990 Workshop on Volume Visualization, ACM, New York, NY, USA, VVS '90, 63-70.

VASILAKIS, A., AND FudOS, I. 2012. S-buffer: Sparsityaware Multi-fragment Rendering. In Eurographics (short papers), Citeseer, 101-104.

Vasilakis, A. A., AND Fudos, I. 2013. Depth-Fighting Aware Methods for Multifragment Rendering. IEEE Transactions on Visualization and Computer Graphics 19, 6 (June), 967-977.

Weiler, M., Kraus, M., Merz, M., and Ertl, T. 2003. Hardware-Based Ray Casting for Tetrahedral Meshes. In Proceedings of the 14th IEEE Visualization 2003 (VIS'03), IEEE Computer Society, Washington, DC, USA, VIS '03, 44-.

Wylie, B., Moreland, K., Fisk, L. A., and Crossno, P. 2002. Tetrahedral Projection Using Vertex Shaders. In Proceedings of the 2002 IEEE Symposium on Volume Visualization and Graphics, IEEE Press, Piscataway, NJ, USA, VVS '02, 7-12.

Yang, J. C., Hensley, J., Grn, H., And Thibieroz, N. 2010. Real-time Concurrent Linked List Construction on the GPU. In Proceedings of the 21st Eurographics Conference on Rendering, Eurographics Association, Aire-la-Ville, Switzerland, Switzerland, EGSR'10, 1297-1304.

Zhao, K., Sakamoto, N., Koyamada, K., Tanaka, S., MUrotani, K., AND KoshizUKA, S. 2015. Volume Rendering for 3D Scattered Data with Interactive Particle-Based Rendering. In Proceedings of International Conference on Systems Simulation (AsiaSim2015). 